\section{Prevalence of joint pain is higher among women in rural Japan than urban Japanese-American women in Hawaii}

\author{
Kiyoshi Aoyagi, Philip D Ross, Chun Huang, Richard D Wasnich, Takuo Hayashi, \\ Tai-ichiro Takemoto
}

\begin{abstract}
Objective-Environmental factors such as farming contribute to the frequency of joint symptoms. The purpose of this study is to explore the possible role of environment (lifestyle), by comparing the prevalence of joint pain between Japanese in a rural farming district in Japan and in urban Hawaii.
\end{abstract}

Subjects and Methods-Current or previous pain at specific joints was surveyed among 222 women in rural Japan and 638 Japanese women in urban Hawaii aged 60-79. The age adjusted prevalence was compared using logistic regression.

Results-The prevalence of pain at one or more joints was approximately $70 \%$ in Japan and $50 \%$ in Hawaii. The prevalence of knee pain in Japan ranged from $36 \%$ at ages $60-69$ years to $53 \%$ at $70-79$ years (mean $41 \%$ ), whereas knee pain affected only $20 \%$ of women in Hawaii in both age groups. The odds ratio (and $95 \%$ CI) was $3.2(2.1,4.8)$ for knee pain, and $4.0(2.2,7.4)$ for mid-back pain in Japan, compared with Hawaii. Pain was also significantly more common in Japan at the shoulder, elbow, and ankle, but not at other joints. Women in Japan were shorter and weighed less than in Hawaii. Adjustment for body mass index increased the odds ratios to $4.4(2.9,6.8)$ for knee, and $4.5(2.4,8.5)$ for mid-back pain. Conclusion-Although the potential influence of cultural factors or other sources of bias cannot be ruled out, the large differences in the prevalence of pain at specific joints suggest that environmental factors are probably responsible, because both populations are of similar genetic stock. (Ann Rheum Dis 1999;58:315-319)

Hawaii Osteoporosis Foundation, Honolulu, HI

P D Ross

C Huang

R D Wasnich

\section{Department of}

Orthopaedic Surgery,

Mitsugi Public General

Hospital, Japan

T Hayashi

Correspondence to:

Dr K Aoyagi, Department of

Public Health, Nagasaki

University, School of

Medicine, 1-12-4 Sakamoto,

Nagasaki 852-8523, Japan.

Accepted for publication based studies show that physical disability is
31 December 1998
Both environmental and genetic factors may contribute to the development of joint pain. For example, previous studies have suggested that farming contributes to increased frequency of joint symptoms, ${ }^{6-9}$ and that being overweight is also associated with increased prevalence of joint pain. ${ }^{10-12}$ Other studies have reported associations of genetic markers with osteoarthritis, which is an important source of joint symptoms in the elderly. ${ }^{13}{ }^{14}$ One valuable approach for evaluating the influence of environmental factors, independent of genetic factors, is to study migrant populations. ${ }^{15-17}$ Changes in the lifestyles of the migrant population can be examined for clues that may help explain differences in disease occurrence, relative to the nonmigrant, indigenous population. ${ }^{18}$ frequency of joint pain within specific populations, ${ }^{12} 1920$ but relatively few comparisons between populations. ${ }^{21}$ If obvious differences in the prevalence of joint symptoms between populations can be demonstrated, this may help guide the discovery of important aetiological factors. In this study, we compare the frequency of pain at specific joints among Japanese women in a rural farming district in Japan with that among urban/suburban Japanese-American women in Hawaii, adjusting for age and body mass index.

\section{Methods}

STUDY SAMPLE

The Mitsugi Bone and Joint Study (MBJS) was conducted between 1994 and 1995 on community dwelling people aged $40-85$ in a rural area (Mitsugi town, near Hiroshima, Japan). The town of Mitsugi has a population of approximately 8400 , in which the population of women aged 40 and over is 2600 . Mitsugi is
There have been numerous studies of the a farming district, where many people less than 70 years of age continue to grow rice and vegetables by manual labour, sometimes using machinery. All community dwelling people aged 40 and over were invited by the local government to participate in the general health examinations (annual examinations mandated by the Japanese Health and Medical Services Law for the Elderly). The MBJS was incorporated into the examinations being conducted in Mitsugi Public General Hospital, which is one of the seven general health examination sites in Mitsugi. Participation was not restricted on the basis of geography; people were allowed to visit whichever single examination site was most convenient. The participation rate in Mitsugi was $70 \%$, and a total of 384 women particiNutrition Examination Survey (NHANES I), reported musculoskeletal symptoms crease in prevalence with increasing age. The most frequent site of symptoms was the back, followed by the knee. problems among the elderly ${ }^{3-5}$ According to data from the US National Health and

Joint pain is a very common complaint among the elderly, ${ }^{12}$ which may lead to function ment the elderly constitute a growing segand disability among the aged have become major public health concerns. Population- 
Table 1 Characteristics of the subjects

\begin{tabular}{|c|c|c|c|c|}
\hline \multirow[b]{2}{*}{ Characteristic } & \multicolumn{2}{|c|}{ Native fapanese in rural fapan } & \multicolumn{2}{|c|}{$\begin{array}{l}\text { Fapanese-Americans in urban } \\
\text { Hawaii }\end{array}$} \\
\hline & Range & Median & Range & Median \\
\hline Age & $60-79$ & 67 & $60-79$ & 73 \\
\hline Height $(\mathrm{cm})$ & $132.1-163.3$ & 149.2 & $112.8-169.0$ & 150.8 \\
\hline Weight $(\mathrm{kg})$ & $32.6-78.0$ & 50.4 & $32.7-80.7$ & 52.7 \\
\hline Body mass index $\left(\mathrm{kg} / \mathrm{m}^{2}\right)$ & $15.2-32.1$ & 22.7 & $15.4-53.8$ & 23.1 \\
\hline
\end{tabular}

pated in the MBJS. All subjects gave informed consent before the examination.

The sample of Japanese-Americans living in Hawaii were 726 women aged 55-93, who participated in the Hawaii Osteoporosis Study (HOS) Examination 8, conducted between 1992 and 1994. Most HOS participants live in the city of Honolulu or its suburbs. Details concerning recruitment and examination of the HOS subjects have been described elsewhere. ${ }^{162}$ Briefly, male subjects of the HOS were recruited from the Honolulu Heart Program (HHP), which is a prospective cohort study of coronary heart disease and stroke among men of Japanese ancestry born between 1900-1911 and living on the island of Oahu, Hawaii in 1965. Using the World War II Selective Service Roster, 11148 eligible men were identified, and a total of 8006 men participated in the first HHP examination during 1965-1968. In 1980, a 30\% random sample of the men attending the third HHP examination, and their wives, if also of Japanese ancestry, were invited to participate in the HOS. A total of 1379 men and 1105 wives participated in the first HOS examination during 1981-1982. Subsequent examinations have been conducted at intervals of one to three years. The wives were the focus of the eighth HOS examination, which included a survey of joint pain. The participation rate among surviving women at the eighth HOS examination was $77 \%$. All subjects gave informed consent before each examination.

\section{MEASUREMENTS}

Information on painful joints was collected by questionnaire; the same questionnaire (except for language) was used to survey both populations so that valid comparisons could be made. The wording of the question was administered in both Japanese and English in the Hawaii study. The original questionnaire was written in
English, translated into Japanese by a native Japanese, and back translated by a different person to check its accuracy. The Mitsugi study used only the Japanese wording from the Hawaii study. Subjects were asked "Which of your joints have ever been painful, swollen, aching, or tender on most days for at least one month (currently, or in the past)?" This question was based on the first United States National Health and Nutrition Examination Survey (NHANES I). ${ }^{1}$ Response boxes (yes and no) for both sides of the body were provided on an illustration of the skeleton for each joint (shoulders, elbows, wrists, hands/fingers, hips, knees, ankles, feet, neck, upper back, mid-back, and low back). Height and weight were measured with the subjects in light clothing and without shoes. Body mass index (BMI) was calculated as weight $(\mathrm{kg}) /$ height $(\mathrm{m})^{2}$.

\section{STATISTICAL ANALYSIS}

Student's $t$ test was used to evaluate statistical significance when comparing the characteristics of the subjects between Japan and Hawaii. The $\chi^{2}$ test was used to evaluate differences between groups for categorical variables. Logistic regression was used to evaluate differences in the prevalence of specific joint pain between the two populations, adjusting for age and BMI (both as continuous variables), including tests for interaction. SAS software (Cary, NC) was used for the data analysis. Analyses were limited to the 222 women in Japan and 638 women in Hawaii aged 60 to 79 , to provide a comparable age range for both populations.

\section{Results}

CHARACTERISTICS OF PARTICIPANTS

Table 1 shows the characteristics of participants in Japan and Hawaii. The median age of women in Hawaii was six years greater than women in Japan, and they had significantly greater body size (height, weight) and BMI than the native Japanese women $(\mathrm{p}<0.05)$.

PREVALENCE OF JOINT PAIN

The prevalence of pain at one or more joints was approximately $72 \%$ in Japan and $51 \%$ in Hawaii. The prevalence did not vary appreciably with age in either community, except for any joint pain and knee pain in Japan, which were significantly higher in the 70-79 year age group than the 60-69 year olds (table 2). Knee,

Table 2 Frequency (\%) of women with joint pain in rural fapan and urban Hawaii

\begin{tabular}{|c|c|c|c|c|c|c|}
\hline \multirow[b]{2}{*}{ Age group (y) } & \multicolumn{3}{|l|}{ Rural fapan } & \multicolumn{3}{|c|}{ Urban Hawaii } \\
\hline & $60-69$ & $70-79$ & Total & $60-69$ & $70-79$ & Total \\
\hline Number of subjects & 154 & 68 & 222 & 135 & 503 & 638 \\
\hline Any joint pain & $103(66.9)$ & $57(83.8)^{\star}$ & $160(72.1)$ & $65(48.2)$ & $257(51.1)$ & $322(50.5)$ \\
\hline Shoulder & 43 (27.9) & $16(23.5)$ & $59(26.6)$ & $23(17.0)$ & $107(21.3)$ & $130(20.4)$ \\
\hline Elbow & $20(13.0)$ & $5(7.4)$ & $25(11.3)$ & $9(6.7)$ & $21(4.2)$ & $30(4.7)$ \\
\hline Wrist & $12(7.8)$ & $8(11.8)$ & $20(9.0)$ & $9(6.7)$ & $23(4.6)$ & $32(5.0)$ \\
\hline Hand & $20(13.0)$ & $10(14.7)$ & $30(13.5)$ & $25(18.5)$ & $90(17.9)$ & $115(18.0)$ \\
\hline Hip & $15(9.7)$ & $6(8.8)$ & $21(9.5)$ & $12(8.9)$ & $53(10.5)$ & $65(10.2)$ \\
\hline Knee & $56(36.4)$ & $36(52.9)^{\star}$ & $92(41.4)$ & $27(20.0)$ & $107(21.3)$ & $134(21.0)$ \\
\hline Ankle & $18(11.7)$ & $11(16.2)$ & $29(13.1)$ & $14(10.4)$ & $31(6.2)$ & $45(7.1)$ \\
\hline Foot & $14(9.1)$ & $9(13.2)$ & $23(10.4)$ & $16(11.9)$ & $48(9.5)$ & $64(10.0)$ \\
\hline Neck & $26(16.9)$ & $12(17.7)$ & $38(17.1)$ & $19(14.1)$ & $71(14.1)$ & $90(14.1)$ \\
\hline Upper back & $19(12.3)$ & $7(10.3)$ & $26(11.7)$ & $12(8.9)$ & $39(7.8)$ & $51(8.0)$ \\
\hline Mid-back & $21(13.6)$ & $13(19.1)$ & $34(15.3)$ & $5(3.7)$ & $28(5.6)$ & $33(5.2)$ \\
\hline Low back & $38(24.7)$ & $23(33.8)$ & $61(27.5)$ & $28(20.7)$ & $116(23.1)$ & $144(22.6)$ \\
\hline
\end{tabular}

${ }^{\star} \mathrm{p}<0.05$ compared with $60-69$ age group. 
Table 3 Odds ratios (95\% confidence intervals) of joint pain comparing women in rural fapan with women in urban Hawaii

\begin{tabular}{lll}
\hline & Age adjusted & \multicolumn{2}{l}{ Age and BMI adjusted } \\
\hline Any joint pain & $2.91(1.97,4.30)$ & $3.41(2.27,5.13)$ \\
Shoulder & $1.58(1.03,2.41)$ & $1.81(1.16,2.81)$ \\
Elbow & $2.27(1.15,4.46)$ & $2.48(1.23,5.00)$ \\
Wrist & $1.98(0.98,3.99)$ & $2.11(1.02,4.36)$ \\
Hand & $0.70(0.42,1.16)$ & $0.77(0.46,1.29)$ \\
Hip & $0.93(0.51,1.71)$ & $1.07(0.57,2.02)$ \\
Knee & $3.20(2.14,4.77)$ & $4.38(2.85,6.75)$ \\
Ankle & $1.97(1.08,3.57)$ & $2.42(1.30,4.51)$ \\
Foot & $0.99(0.55,1.80)$ & $1.19(0.64,2.21)$ \\
Neck & $1.25(0.77,2.05)$ & $1.43(0.86,2.39)$ \\
Upper back & $1.38(0.75,2.51)$ & $1.51(0.81,2.80)$ \\
Mid-back & $4.02(2.18,7.43)$ & $4.48(2.38,8.46)$ \\
Low back & $1.44(0.95,2.18)$ & $1.83(1.19,2.83)$ \\
\hline
\end{tabular}

$\mathrm{BMI}=$ body mass index. Odds ratios were estimated by logistic regression, adjusting for age and BMI (continuous variables).

low back and shoulder pain were the most prevalent locations of joint pain in both Japan and Hawaii, with at least $20 \%$ of the women affected in both populations. In Japan, the prevalence of knee pain increased with age; ranging from $36 \%$ in $60-69$ years to $53 \%$ in 70-79 years (crude prevalence of $41 \%$ for all ages combined), whereas knee pain affected only $20 \%$ to $21 \%$ of women in Hawaii in both age groups. The prevalence of shoulder pain did not vary consistently among age groups in either community. The prevalence of low back pain increased slightly with age from approximately $25 \%$ to $34 \%$ in Japan, whereas it did not vary meaningfully in Hawaii $(21 \%$ to $23 \%)$ over same age range.

COMPARISON OF JOINT PAIN BETWEEN RURAL JAPAN AND URBAN HAWAII

The odds ratios (OR) and 95\% confidence intervals (CI) comparing joint pain among the women in Japan to those in Hawaii, with adjustment for age and BMI, are shown in table 3. The age adjusted OR for knee pain was 3.2, which increased to 4.4 after additional adjustment for BMI. The adjusted prevalence of mid-back pain was higher in Japan than in Hawaii (age adjusted OR $=4.0$ ) whereas the OR for neck, upper back, and low back pain were smaller (1.3 to 1.4 ). Pain was also significantly more common in Japan at the shoulder, elbow and ankle, but not at other joints. Additional adjustment for BMI increased the magnitudes of associations somewhat, and the associations for wrist and lower back pain became significant as a result.

\section{Discussion}

Comparing migrant populations with residents of the country of origin may help to identify risk factors responsible for observed differences in disease, by taking advantage of the increased variability in environmental factors while limiting genetic differences. ${ }^{151618}$ It is likely that Japanese-Americans in Hawaii are of similar genetic stock to native Japanese. For most of its history, Japan had relatively little contact with the rest of the world, and its genetic pool is relatively homogeneous. ${ }^{23}$ Thus, our results suggest that environmental factors (rural compared with urban) may be responsible for the large differences in the frequency of pain at certain joints. The finding that differences are limited to certain joints suggests potential opportunities to identify aetiological factors.

Previous studies have reported that psychosocial factors (including those related to occupation) are associated with reporting of joint pain. ${ }^{24}$ Information about occupation was not available for the women in our study. However, Mitsugi is primarily a farming district, whereas Hawaii is an urban/suburban setting. Many women in Mitsugi work at farming until about age 70 years, whereas many of the women in Hawaii are either housewives, or retire from the workforce at age 65 years, or earlier. Thus, the differences in frequency of joint pain reported here may be partly attributable to occupational differences such as current employment, length of employment, type of labour, or psychosocial factors.

Cultural differences other than occupation may also affect the propensity to report symptoms, and may be partly responsible for the observed differences in prevalence of joint pain. Differences in social stigmas and stoicism between Japan and Hawaii might also partly explain differences in reported joint pain rather than biological differences in diseases such as osteoarthritis (OA) or fractures. However, one would expect such biases to be uniform for all joints. The observed joint specific differences therefore suggest that such cultural characteristics are probably not important in this regard.

Mitsugi town is in the same prefecture as the city of Hiroshima, but is distinctly different from Hiroshima city, where the atomic bomb was exploded. Although most people in our study population in Japan did not suffer direct physical consequences from the atomic bomb, they may have suffered emotionally. Furthermore, hardships including shortages of food, medicines, and other supplies both during and after the war were probably felt more in Mitsugi than in Hawaii. This is another factor that may partly explain differences in joint pain relative to Hawaii.

In this study, we used logistic regression to compare the prevalence of pain at specific joints between the two populations, adjusting for age and BMI. The effect of adjustment for BMI was to increase the association of joint pain with living in Mitsugi. Although BMI was associated with joint pain in this study, and obesity is a risk factor for knee OA and joint pain in other populations, ${ }^{1026}$ differences in obesity seem to be less important than other (as yet unknown) risk factors for explaining differences in joint pain prevalence between Japan and Hawaii.

An advantage of logistic regression is the ability to control for differences in age and other potential confounders. However, the resulting odds ratios will overestimate the true association when the outcome of interest is greater than $5-10 \%$. Thus, the relative prevalence of joint pain is not as high as suggested by the odds ratios in this study, because of the high prevalence of joint pain. Furthermore, adjustment for age in the logistic regression assumes that the association between age and joint pain 
is similar in magnitude for both populations. There is some evidence that the prevalence of pain increases with age in Japan but not in Hawaii, as noted earlier, and this may have influenced the estimated odds ratios for knee pain and for all joints combined. However, there were no significant interactions between age and country of residence in the logistic regression models. For these comparisons, the reader can also compare the age specific prevalence values provided in table 2 , which are not affected by the logistic regression model assumptions.

The identification of painful joint(s) was based on subjects' responses to the question: "Which of your joints have ever been painful, swollen, aching, or tender on most days for at least one month (currently, or in the past)?" There is substantial overlap between this question and the criteria for the classification and clinical diagnosis of OA. Although there is correlation between clinical signs of $\mathrm{OA}$ and radiographic OA, clinical signs are often present without radiographic evidence and moderate and severe radiographic OA is often present without clinical signs. ${ }^{27}$ The Subcommittee on Osteoarthritis of the American College of Rheumatology's Diagnostic and Therapeutic Criteria Committee selected "joint pain for most days of the prior month" as a major inclusion parameter for identifying clinical OA of the hand, hip and knee. ${ }^{28} \mathrm{~A}$ similar question has been used for identifying symptomatic OA of the knee. ${ }^{29-31}$ Although OA is not the only musculoskeletal disease contributing to joint pain, it is the most common type of joint disease in geriatric patients. ${ }^{32}$ Thus, joint pain may partly represent symptomatic OA for certain joint groups such as knee, hip, and hand in the current study.

This study has several limitations. Radiographs were not available for diagnosis of OA or for diagnosis of fractures in the Mitsugi group. We cannot prove whether the observed differences in body size existed before the occurrence of joint pain, because of the cross sectional design of the study. Information on other possible risk factors for joint pain such as history of joint injuries, ${ }^{11}$ occupation, etc, were not available. Possible differences in the selection of subjects between Japan and Hawaii are potential sources of bias. For example, the Japanese who migrated to Hawaii may have carried genetic traits that could influence the musculoskeletal characteristics of their children differently than those who remained in Japan. It was not possible to determine either the existence or magnitude of such effects in this study, but comparisons of blood group patterns suggest that genetic differences may be small. ${ }^{15}$ Another issue is different types of sampling between Japan and Hawaii, which could affect the results. For example, the women in Japan were participants in general health examinations by the local government, and the women in Hawaii were wives of men who had participated in previous epidemiological studies. Although $98 \%$ of JapaneseAmericans in this age range have been married, and fewer than $1 \%$ have been divorced, the subjects in this study may have not necessarily been representative of the general population. To minimise such potential sources of bias, future studies that intend to compare populations should make every possible effort to select persons randomly from a defined base population.

In summary, this study suggests that important differences among women aged 60-79 exist between Japan and Hawaii in the observed prevalence of joint pain, and may be related to differences in environmental factors. Further comparisons between populations may help to identify the responsible factors, which might be modified to reduce the burdens of joint pain and associated disability.

We would like to thank Dr Noboru Yamaguchi (Mitsugi Public General Hospital) for his generous support of the study, and also thank the staff of Mitsugi Public General Hospital and Mitsugi Health and Welfare Center for their valuable assistance Mitsugi Health and Welfare Center for their valuable assistance
in conducting the study. The study was supported in part by the in conducting the study. The study was supported in part by the
National Institutes of Health, National Institute on Aging (grant \#AG10412) and Hawaii Osteoporosis Foundation

1 Cunningham LS, Kelsey JL. Epidemiology of musculoskeletal impairments and associated disability. Am J Public etal impairments and

2 Miles TP, Flegal K, Harris T. Musculoskeletal disorders: time trends, comorbid conditions, self-assessed health status, and associated activity limitation. Vital Health Stat 3 1993;27:275-88

3 Reynolds DL, Chambers LW, Badley EM, Bennett KJ, Goldsmith $\mathrm{CH}$, Jamieson E, et al. Physical disability among Canadians reporting musculoskeletal diseases. J Rheumatol 1992;19:1020-30.

4 Hubert HB, Bloch DA, Fries JF. Risk factors for physical disability in an aging cohort: the NHANES I Epidemiologic Followup Study. J Rheumatol 1993;20:480-8.

5 Badley EM, Tennant A. Impact of disablement due to rheumatic disorders in a British population: estimates of severity and prevalence from the Calderdale Rheumatic Disablement Survey. Ann Rheum Dis 1993;52:6-13.

6 Maeda K, Okazaki F, Suenaga T, Sakurai T, Takamatsu M. Low back pain related to bowing posture of greenhouse Low back pain related to bowing post
farmers. J Hum Ergol 1980;9:117-23.

7 Almas R, Odegard J. Morbidity among self-employed farmers in Norway. Scand J Soc Med 1985;13:169-72.

8 Leigh JP, Sheetz RM. Prevalence of back pain among fulltime United States workers. Br J Ind Med 1989;46: 651-7.

9 Schenker MB. Preventive medicine and health promotion are overdue in the agricultural workplace. J Public Health Policy 1996;17:275-305.

10 Aro S, Leino P. Overweight and musculoskeletal morbidity: a ten-year follow-up. Int J Obes 1985;9:267-75.

11 Wigley RD, Prior IA, Salmond C, Stanley D, Pinfold B. Rheumatic complaints in Tokelau. I. Migrants resident in New Zealand. The Tokelau Island migrant study. Rheumatol Int 1987;7:53-9.

12 Huang C, Ross PD, Lydick E, Wasnich RD. Factors associated with joint pain among postmenopausal women. Int $\mathrm{J}$ Obes 1997;21:349-54.

13 Uitterlinden AG, Burger H, Huang Q, Odding E, Duijn $\mathrm{CM}$, Hofman A, et al. Vitamin D receptor genotype is associated with radiographic osteoarthritis at the knee. J Clin Invest 1997;100:259-63.

14 Cicuttini FM, Spector TD. Genetics of osteoarthritis. Ann Rheum Dis 1996;55:665-7.

15 Kagan A, Harris BR, Winkelstein W Jr, Johnson KG, Kato $\mathrm{H}$, Syme SL, et al. Epidemiologic studies of coronary heart disease and stroke in Japanese men living in Japan, Hawail disease and sto: and California: demographic, physical, dietary and bio-

16 chemical characteristics. J Chronic Dis 1974;27:345-64. Heilbrun LK, Kagan A, Nomura A, Wasnich RD. The origins of epidemiologic studies of heart disease, cancer and
osteoporosis among Hawaii Japanese. Hawaii Med J 1985; 44:294-6.

17 Spector TD, Hochberg MC. Methodological problems in the epidemiological study of osteoarthritis. Ann Rheum Dis 1994;53:143-6.

18 Ross PD, Orimo H, Wasnich RD, Vogel JM, MacLean CJ, Davis JW, et al. Methodological issues in comparing genetic and environmental influences on bone mass. Bone Miner 1989;7:67-77.

19 Lawrence RC, Hochberg MC, Kelsey JL, McDuffie FC, Medsger TA Jr, Felts WR, et al. Estimates of the prevalence of selected arthritic and musculoskeletal diseases in the United States. J Rheumatol 1989;16:427-41.

20 Andersson HI, Ejlertsson G, Leden I, Rosenberg C. Chronic pain in a geographically defined general population: studies of differences in age, gender, social population: studies of differences in age, gender, social

21 Hameed K, Gibson T. A comparison of the prevalence of rheumatoid arthritis and other rheumatic diseases amongs Pakistanis living in England and Pakistan. Br J Rheumato 1997;36:781-5. 
22 Worth RM, Kagan A. Ascertainment of men of Japanese ancestry in Hawaii through World War II Selective Service registration. J Chronic Dis 1970;23:389-97.

23 Ross PD, Norimatsu H, Davis JW, Yano K, Wasnich RD, Fujiwara $\mathrm{S}$, et al. A comparison of hip fracture incidence among native Japanese, Japanese Americans, and American Caucasians. Am J Epidemiol 1991;133:801-9.

24 Bongers PM, de Winter CR, Kompier MA, Hildebrandt VH. Psychosocial factors at work and musculoskeletal disease. Scand J Work Environ Health 1993;19:297-312.

25 Hales TR, Bernard BP. Epidemiology of work-related musculoskeletal disorders. Orthop Clin North Am 1996;27: 679-709.

26 Felson DT, Anderson JJ, Naimark A, Walker AM, Meenan RF. Obesity and knee osteoarthritis. The Framingham Study. Ann Intern Med 1988;109:18-24.

27 Bagge E, Bjelle A, Eden S, Svanborg A. Osteoarthritis in the elderly: clinical and radiological findings in 79 and 85 year olds. Ann Rheum Dis 1991;50:535-9.
28 Silman AJ, Hochberg MC. Osteoarthritis. In: Epidemiology of the rheumatic diseases. Oxford: Oxford University Press, 1993:257-88.

29 Felson DT, Naimark A, Anderson J, Kazis L, Castelli W, Meenan RF. The prevalence of knee osteoarthritis in the elderly. The Framingham Osteoarthritis Study. Arthritis Rheum 1987;30:914-18.

30 Anderson JJ, Felson DT. Factors associated with osteoarthritis of the knee in the first national Health and Nutrition Examination Survey (HANES I). Evidence for an association with overweight, race, and physical demands of work. Am J Epidemiol 1988;128:179-89.

31 Felson DT, Zhang Y, Anthony JM, Naimark A, Anderson JJ. Weight loss reduces the risk for symptomatic knee osteoarthritis in women. The Framingham Study. Ann Intern Med 1992;1 16:535-9.

32 Calkins E. Arthritis in the elderly. Bull Rheum Dis 1991;40: $1-9$. 